\title{
EFFECT OF CARBON NANOTUBE INTERFACIAL GEOMETRY ON THERMAL TRANSPORT IN SOLID-LIQUID PHASE CHANGE MATERIALS
}

\author{
Ronald J Warzoha ${ }^{\mathrm{a}}$ and Amy S Fleischer ${ }^{\mathrm{b},{ }^{* 1}}$ \\ ${ }^{a}$ Department of Mechanical Engineering, United States Naval Academy, Annapolis, MD 21402 \\ ${ }^{\mathrm{b}}$ Department of Mechanical Engineering, Villanova University, Villanova, PA 19085
}

\section{Abstract}

For nearly two decades, research groups have attempted to increase the thermal conductivity of bulk materials by saturating them with different concentrations and types of nanoparticles. However, optimal enhancements in the thermal conductivity of these materials continue to go unrealized, primarily due to interfacial phenomena that impede phonon propagation from the bulk material to the nanoparticle or between individual, contacting nanoparticles. Though it is almost certain that interfacial resistances between contacting nanopartilces are responsible for the underwhelming thermal performance of nanoparticles in bulk materials, the physical mechanisms that limit thermal transport at nanoparticle junctions is not well understood. In this study, we investigate the effect of nanoparticle constriction size (i.e. the contact area between linked nanoparticles) on the bulk thermal conductivity of a surrounding paraffin-based phase change material. To this end, we measure the effective thermal conductivity of different Multiwalled Carbon Nanotube (MWCNT)/paraffin nanocomposites is measured with the transient plane source technique. Using a newly developed physical model, the interfacial thermal resistance between the contacting nanoparticles is determined as a function of the constriction geometry. Results suggest that the thermal conductance (i.e. the rate of heat transfer) between contacting MWCNTs can be increased by a factor of 27 by increasing their diameter by only 58 $\mathrm{nm}$. This is in direct conflict with effective medium approximations, which predict an increase in the bulk thermal conductivity of MWCNT-PCM composites when MWCNT diameters are reduced. Such a result indicates that the contact area between individual, contacting nanoparticles has a significant impact on thermal transport within the PCM nanocomposite, and therefore its bulk thermal conductivity. It is expected that these results will strongly impact the design of nanoparticle-laden PCMs.

\footnotetext{
${ }^{1}$ Corresponding Author, e-mail: amy.fleischer@villanova.edu
} 
33 Key Words: Phase change materials; multi-walled carbon nanotubes; phonon; thermal conductivity; constriction resistance; thermal boundary conductance

\section{Introduction}

37 Organic phase change materials (PCMs) have the ability to remove and store heat passively, thereby making them excellent candidates for the thermal management of portable electronic devices [1] and electric vehicle batteries [2], as well as for storing and reusing thermal energy in residential solar-thermal and HVAC systems [3]-[5]. The amount of heat that can be stored within a PCM is governed by its phase change enthalpy, which is typically high for commercial paraffin waxes (on the order of $200 \mathrm{~kJ} / \mathrm{kg}$ [6], [7]). As a result of their high phase change enthalpies, the amount of heat that can be stored in paraffins on a per unit mass basis is expected to be sufficient for meeting the performance expectations of the aforementioned applications.

Unfortunately, paraffin-based PCMs have exceptionally low thermal conductivities (on the order of $0.1 \mathrm{~W} / \mathrm{mK}[8])$. This is over four orders of magnitude lower than the thermal conductivity of conventional heat transfer materials (oxygen-free copper, for instance, has a thermal conductivity of $\sim 400 \mathrm{~W} / \mathrm{mK}[9])$. The poor thermal conductivities of organic PCMs result in extremely slow melt and solidification rates and thus very little utilization of the PCM mass in thermal energy storage applications [6], [7], [10]. Low PCM thermal conductivities can also result in severe thermal gradients in close proximity to sensitive electronics and thus failure of the primary electronic component(s) in critical energy infrastructure. Consequently, it is imperative that the thermal conductivity of organic PCMs be increased.

In this work, multi-walled carbon nanotubes (MWCNTs) are inserted into an organic paraffin PCM in order to improve its bulk thermal conductivity. MWCNTs are known to have remarkably high intrinsic thermal conductivities (as high as 6,000 W/mK [11]), low densities and high surface area-to-volume ratios, making them excellent candidates for PCM thermal enhancement

60 in applications with weight and volume constraints. Additionally, the thermal conductivity of

61 MWCNTs can be nearly an order of magnitude greater than some of the conventional materials used in thermal management systems, primarily due to the strong $\mathrm{sp}^{2}$ bonds that are formed

63 between neighboring carbon atoms and the long mean free path of phonons within the 64 nanoparticle itself [12], [13] ( 1 $\mu \mathrm{m}$ for CNTs and graphene [14]). 
However, the incorporation of nanoparticles into PCMs for thermal augmentation is not straightforward. This is primarily due to the fact that standard effective medium approximations often over predict the thermal conductivity of nanocomposite materials. Instead, the thermal conductivity enhancement of a nanocomposite is generally inhibited by a thermal resistance that occurs across nanoparticle interfaces [15]. This thermal resistance is governed by a variety of geometric, physical and chemical phenomena that occur at the nanoparticle-nanoparticle junction(s), and can be substantial when the nanoparticles have rough surfaces or extend in 73 multiple dimensions [14] (as high as $10^{-4} \mathrm{~m}^{2} \mathrm{~K} / \mathrm{W}$ for carbon nanofibers, for instance [6]). On the

74 other hand, recent molecular dynamics simulations suggest that the presence of carbon-based nanoparticles with 'smooth' surfaces (such as CNTs and graphene) results in the alignment of alkane molecules across carbon nanoparticle-paraffin interfaces. In turn, a significant reduction in the interfacial thermal resistance at this type of interface can be achieved [16]. Consequently, the use of carbon-based nanoparticles for enhancing the thermal conductivity a paraffin-based PCM offers the most promising method for their thermal conductivity enhancement to date.

Several groups have decreased the thermal resistance at nanoparticle-nanoparticle junctions by increasing the adhesion energy across contacting interfaces. This technique generally results in mechanically strong, percolating networks [17]-[20]. In these studies, assortments of chemical modifications are made at the nanoparticle junction(s) in order to achieve higher rates of thermal transport and increased mechanical stability. However, it is not known whether the thermal conductivity of these percolating nanocomposite PCMs can be further tuned by adjusting the size of the constriction at the interface. Preliminary molecular dynamics simulations suggest that the size of the constriction does affect thermal transport at an individual nanoparticle-nanoparticle junction [21], [22], but the range of nanoparticle sizes used for these predictions is too small to make accurate estimations about its influence on both nano-scale and macro-scale thermal

91 transport within PCMs. Additionally, it is unknown to what degree ballistic and diffusive 92 transport affect heat flow rates at these junctions. As a result, additional work is needed to 93 determine the effect of nanoparticle contact area on the thermal performance and associated 94 nano-scale and macro-scale thermal characteristics of nanocomposite PCMs.

In this study, four different MWCNTs with varying diameters and lengths are embedded within

97 an organic paraffin PCM at concentrations above and below the percolation threshold. The 98 objectives of this study are to determine the effect of CNT contact area on: (1) the interfacial 
99 thermal resistance between a CNT and another CNT and (2) the ballistic and diffusive

100 components of heat transfer on heat flow at the interface. The effective interfacial thermal

101 resistance between two linked CNTs is calculated by saturating the PCM with different volume

102 concentrations of CNTs and applying the effective medium model for thermal conductivity of

103 networked composites developed by Foygel et al. [23]. The procedure for this method is

104 described in [15]. The insight gained from this study will allow for the design of nanocomposite

105 PCMs with tunable thermal conductivity. In this case, thermal engineers will be able to tune the

106 thermal properties of disordered, bulk materials based on the phonon physics that occur at sub-

107 micron CNT interfaces. Such insight will allow for the thermal optimization of next-generation

108 CNT-based composite materials.

109

\section{Experimental and Analytical Methods}

111 Physical Properties of Materials

112 The geometric properties for each of the different nanoparticles that are used in this study are

113 listed in Table 1. In total, four different types of CNTs are used to produce the nanocomposites

114 for this work. The values for the diameter, length and density of the nanoparticles are obtained

115 from the manufacturer (US Research Nanomaterials, Inc.).

116

\begin{tabular}{lcccccc}
\hline $\mathbf{v}$ & $\begin{array}{c}\text { Density } \\
\left(\mathbf{k g} / \mathbf{m}^{\mathbf{3}}\right)\end{array}$ & $\begin{array}{c}\text { Diameter } \\
(\mathbf{n m})\end{array}$ & $\begin{array}{c}\text { Length } \\
(\boldsymbol{\mu m})\end{array}$ & $\begin{array}{c}\text { Aspect } \\
\text { Ratio }\end{array}$ & $\begin{array}{c}\mathbf{\Phi}_{\mathbf{c}} \\
(\boldsymbol{\%})\end{array}$ & $\begin{array}{c}\mathrm{A}_{\mathrm{o}} \\
\left(\mathbf{n m}^{\mathbf{2}}\right)\end{array}$ \\
\hline \hline $\mathbf{1}$ & 2100 & 7 & 20 & 2857.0 & 0.022 & 270 \\
$\mathbf{2}$ & 2100 & 10 & 50 & 5000.0 & 0.012 & 587 \\
$\mathbf{3}$ & 2100 & 25 & 15 & 800.0 & 0.078 & 2390 \\
$\mathbf{4}$ & 2100 & 65 & 15 & 230.8 & 0.269 & 16500 \\
\hline
\end{tabular}

Table 1: MWCNT physical properties

119 In Table $1, \Phi_{c}$ is the volume fraction at which the nanoparticles begin to percolate and $A_{o}$ is the 120 average overlap area between nanoparticles (which is distinctly different from contact area,

121 mentioned later). Eqn. 1, developed by Foygel et al. [23] (and later confirmed by Wemhoff 122 [24]), is used to determine $\Phi_{\mathrm{c}}$.

123

$124 \quad \phi_{c}=\frac{0.6}{p}$ 
126 In Eqn. 1, p represents the aspect ratio of the nanoparticle. The average overlap area between

$129 \quad A_{o}=\frac{2 D^{2}}{\pi} \zeta(p)$

131 where,

$133 \zeta(p)=\ln \left[\frac{\sqrt{1+p^{-1}}+\sqrt{1-p^{-1}}}{\sqrt{1+p^{-1}}-\sqrt{1-p^{-1}}}\right]$

In Eqns. 2 and 3, D is the diameter of the nanotube, and $\mathrm{p}$ is the aspect ratio of the nanoparticle

$136(\mathrm{p}=\mathrm{L} / \mathrm{D}$, where $\mathrm{L}$ is the length of the nanoparticle).

The thermophysical properties of the commercial paraffin (IGI 1230A) used in this work are listed in Table 2 and are supplied by the manufacturer.

\begin{tabular}{cccccc}
\hline $\begin{array}{c}\text { Thermal } \\
\text { Conductivity } \\
(\mathbf{W} / \mathbf{m K})\end{array}$ & $\begin{array}{c}\text { Density } \\
\left(\mathbf{k g} / \mathbf{m}^{3}\right)\end{array}$ & $\begin{array}{c}\text { Specific } \\
\text { Heat }\end{array}$ & $\begin{array}{c}\text { Thermal } \\
\text { Diffusivity } \\
(\mathbf{J} / \mathbf{k g K})\end{array}$ & $\begin{array}{c}\text { Melt } \\
\left(\mathbf{m m}^{2} / \mathbf{s}\right)\end{array}$ & $\begin{array}{c}\text { Phase } \\
\text { Change }\end{array}$ \\
& & & & & $\begin{array}{c}\text { Enthalpy } \\
(\mathbf{k J} / \mathbf{k g})\end{array}$ \\
\hline \hline 0.25 & 880 & 2100 & 0.14 & 329.15 & 290
\end{tabular}

\section{Materials Fabrication}

144 Randomly oriented MWCNT/paraffin nanocomposites are synthesized using a two-step 145 technique. MWCNTs are first embedded within a melted paraffin wax $\left(\mathrm{T}_{\text {melt }}=329.15 \mathrm{~K}\right)$ at 146 concentrations ranging from $0.003 \mathrm{v} \%$ to $5 \mathrm{v} \%$ and are subsequently dispersed using a sonic 147 horn. For dispersion, the ultrasonic horn is pulsed continuously at $32 \mathrm{~Hz}$ for 20 minutes. The 148 resulting liquid paraffin composite material is poured into two separate cylindrical molds 149 (diameter $=2.8 \mathrm{~cm}$, height $=1.4 \mathrm{~cm}$ ) that rest on a polished stainless steel substrate. The mirror 150 finish on the stainless steel substrate is designed to minimize the surface roughness of the face of 151 the solid composite that directly touches the Transient Plane Source sensor in order to minimize 152 the thermal interface resistance between the sensor and the surrounding material (it should be 153 noted, though, that this effect can be eliminated by adjusting the starting time for the Transient 
154 Plane Source curve fit procedure, which is described briefly in the next section and in detail in 155 other work [25], [26]).

Thermal Conductivity Measurements

159 The thermal conductivity of each MWCNT/paraffin nanocomposite is measured using a

160 Transient Plane Source (TPS) device. The details of this measurement technique and its use for 161 measuring the thermal conductivity of nanocomposites are described elsewhere [26]. For this 162 technique, a double-spiral nickel foil sensor is used to both direct heat into a surrounding 163 material and measure the temperature within the foil. The transient temperature distribution 164 within the foil is obtained using a Wheatstone bridge and is related to the temperature rise in a 165 surrounding material as a function of the applied heat flux at the sensor interface. For solid 166 samples, the sensor is sandwiched between each material, while for liquid samples the sensor is 167 immersed within the material. Theoretically, a large temperature rise per unit time corresponds to 168 a more thermally insulating material, whereas a small transient temperature rise indicates that the 169 material behaves as a thermal conductor.

170 A number of different physical phenomena are known to affect the accuracy of this measurement 171 technique. First, it is critical that the thermal penetration depth, $\Delta \mathrm{p}$, does not exceed the distance 172 from the sensor edge to any one of the material's boundaries in order to maintain a semi-infinite 173 boundary condition. The thermal penetration depth can be calculated using Eqn. 4.

$174 \Delta p=2(\alpha \mathrm{t})^{1 / 2}$

175 In Eqn. 4, $\alpha$ represents the thermal diffusivity of the paraffin and t represents the time that the 176 test is run for. In this work, the samples are cylindrical with diameters and heights of $2.8 \mathrm{~cm}$ and

$1771.4 \mathrm{~cm}$, respectively. The maximum thermal penetration depth calculated for any test in this 178 study is $0.8 \mathrm{~cm}$. Given that the radius of the sensor is $3.189 \mathrm{~mm}$, the maximum allowable thermal 179 penetration depth is $\sim 1.08 \mathrm{~cm}$. Thus, all of the samples in this study can be considered semi180 infinite and therefore abide by the geometric constraints of the technique.

181 The accuracy of the TPS method also depends on the length of time that the test is run for. This 182 time is referred to as a characteristic time and can be calculated based on the TPS sensor 183 geometry and the surrounding material's thermal properties $\left(t_{c}=r^{2} / \alpha\right.$, where $r$ is the radius of the 
184 sensor). When the characteristic time is between $0.33 \leq \mathrm{t}_{\mathrm{c}} \leq 1.0$, the solution to the differential

185 equation describing heat flow from the sensor to the surrounding material is insensitive to the

186 curve fitting procedures that are used to relate the data to the solution for the non-linear

187 differential equation [26]. However, significant error results when calculating a solution for the

188 thermal conductivity of the surrounding material if the characteristic time does not fall between

189 these values. Consequently, the characteristic time for all of the samples tested in this work is

190 kept within the range of 0.33 to 1.0 in order to ensure that the solution is accurate.

\section{Calculation of Interfacial Thermal Resistance}

192 The primary objective of this study is to manipulate the bulk thermal conductivity of paraffin

193 PCMs by adjusting the contact area between adjacent, percolating CNTs in order to augment the

194 PCM's melt front progression and solidification rates. To determine the physical mechanisms

195 that are responsible for any enhancement or degradation in the thermal conductivity of the bulk

196 PCM, the microscale physics that govern heat flow at nanoparticle interfaces are analyzed

197 through the calculation of the interfacial thermal resistance at MWCNT-MWCNT junctions. To

198 conduct this analysis, the model developed by Foygel et al. [23] is used to calculate the average

199 contact area between individually touching MWCNTs, the number of MWCNT junctions per

200 unit volume and the effective interfacial thermal resistance at an individual MWCNT-MWCNT 201 interface.

\section{Results and Discussion}

203 For this work, the effective thermal conductivity of the MWCNT/paraffin nanocomposite PCMs

204 is determined as a function of volume fraction when the MWCNTs are fully percolating. The 205 thermal conductivity enhancement of the paraffin $(\eta)$ is given as a function of volume fraction $206 \quad(\square)$ in Fig. 1. 


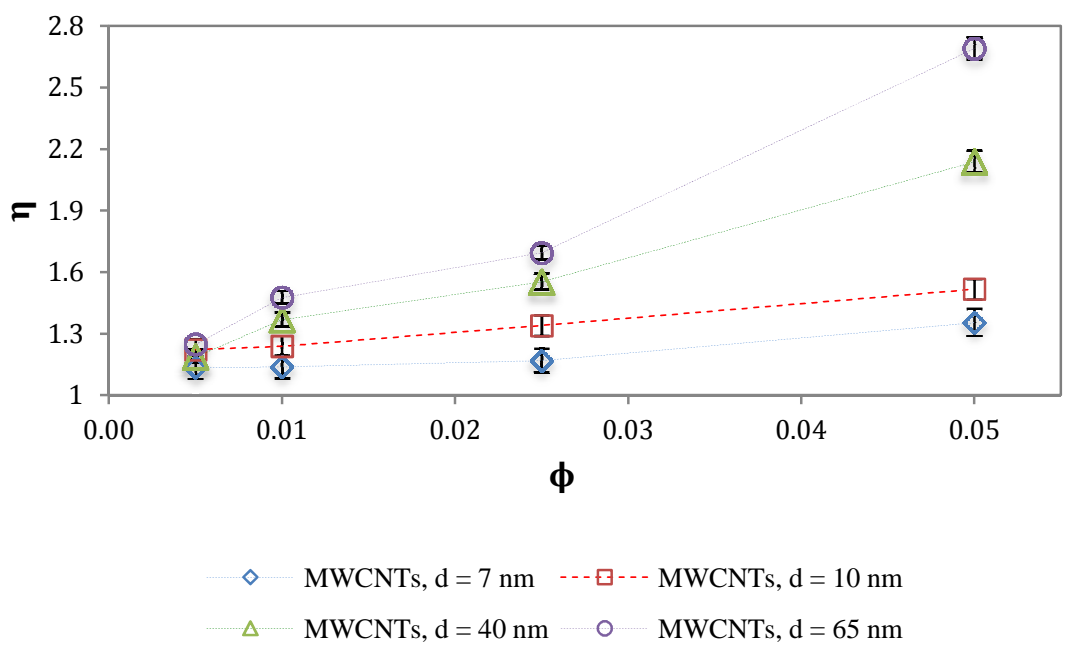

Fig. 1: Thermal conductivity enhancement $(\eta)$ of paraffin

210 As shown in Fig. 1, the bulk thermal conductivity of the MWCNT/paraffin nanocomposites 211 increases with increasing diameter. This result is striking due to the expected nature of thermal 212 transport within the CNTs themselves; as the number of graphene layers surrounding the 213 innermost graphene layer increases (and thus as the diameter of the CNT increases), a greater 214 magnitude of boundary scattering between neighboring graphene layers is expected [12]. 215 Consequently, a reduction in thermal transport within the CNT itself is expected when the 216 number of layers is increased. However, the thermal conductivity enhancement of the 217 MWCNT/paraffin composites is shown to increase with increasing diameter. Thus, simple 218 mixing rules based on the thermal conductivity of the nanoparticles and the surrounding matrix 219 material themselves (represented by Eqn. 5) cannot be used as a basis for the design of 220 MWCNT/paraffin nanocomposites when the nanoparticles are percolating.

$222 k_{e}=k_{p} \phi_{p}+k_{m} \phi_{m}$

224 One possible explanation for this discrepancy is that the flow of heat between nanoparticles is 225 impeded by some interfacial phenomenon. In this case, the only physical parameter that is altered 226 is the contact area between the nanoparticles. Thus, the interfacial thermal resistance between 227 contacting nanoparticles is determined as a function of the average contact area (provided in 228 Table 1) between nanoparticles. For this work, the model developed by Foygel et al. [23] is used 229 to determine the interfacial thermal resistance between nanoparticles as a function of average 230 contact area. 
232 According to Foygel et al. [23], the enhancement in the thermal conductivity of a conventional

233 bulk material is a function of the geometric relationship between the nanoparticle fillers, the

234 morphology of the nanoparticle network(s) and the heat flow rate at contacting nanoparticle

235 junctions when the nanoparticles percolate throughout the material. The effective thermal

236 conductivity $(\sigma)$ is related to these material properties through Eqn. 6.

$238 \sigma(\phi, a)=\sigma_{0}\left(\phi-\phi_{c}\right)^{t(a)}$

240 In Eqn. 6, $\sigma$ represents the effective thermal conductivity of the paraffin nanocomposite, a is the 241 aspect ratio of the nanoparticles embedded within the host material, $\sigma_{0}$ is a preexponential factor 242 that depends on the thermal conductivity and morphology of the contacting nanoparticle 243 networks, $\square_{\mathrm{c}}$ is the volume fraction at which the nanoparticles first begin to form percolating 244 networks and $t(a)$ is a conductivity exponent that depends on the aspect ratio of the nanoparticle.

245 Because there are two unknown parameters in Eqn. 5 ( $\sigma_{0}$ and $\left.t(a)\right)$, an iterative scheme is 246 employed in order to produce a best fit trend to the data obtained for effective thermal 247 conductivity using the TPS technique. These parameters can then be used to solve for the 248 effective interfacial thermal resistance between a set of contacting nanoparticles according to 249 Eqn. 7.

$251 \quad R_{0}=\left(\sigma_{0} \cdot \lambda \cdot \phi_{c}{ }^{t(a)}\right)^{-1}$

253 In Eqn. 7, $\mathrm{R}_{0}$ is the effective interfacial thermal resistance between two contacting MWCNTs and $254 \lambda$ is the average distance between MWCNT junctions. The curve fits for each of the distributions 255 in Fig. 1 are provided in Figs. 2 (a) through (d). 


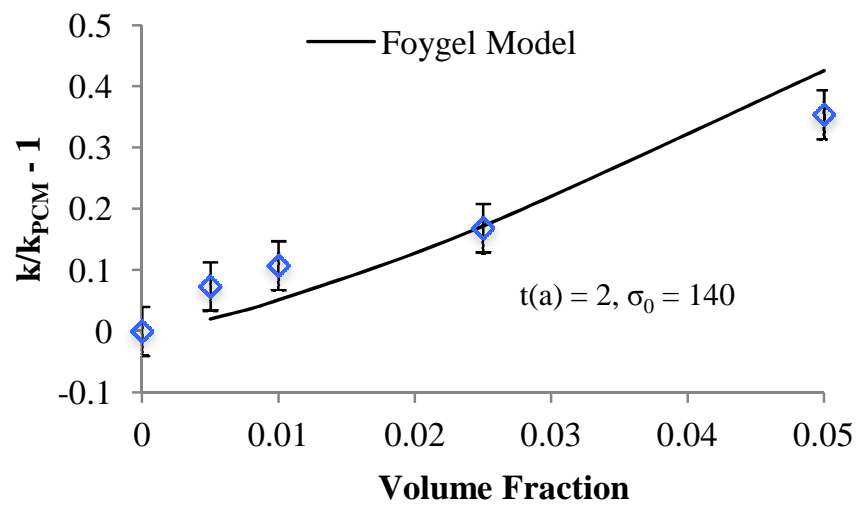

257

258

(a) $7 \mathrm{~nm}$ MWCNTs

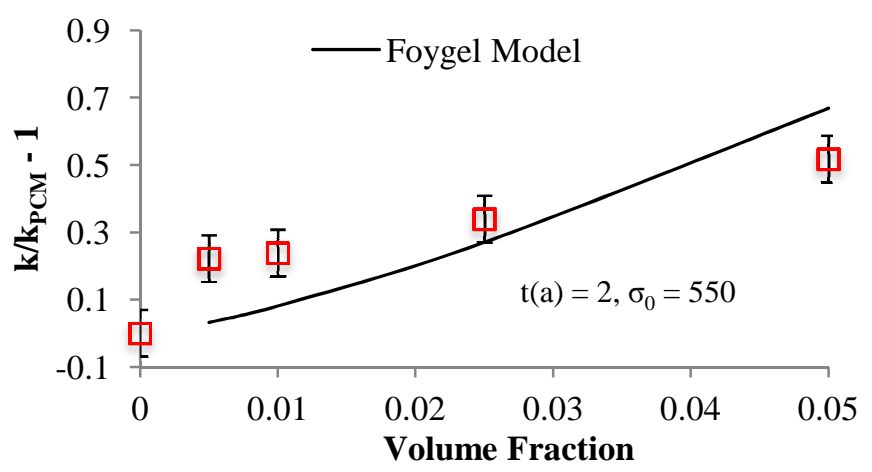

259

260

(b) $10 \mathrm{~nm}$ MWCNTs

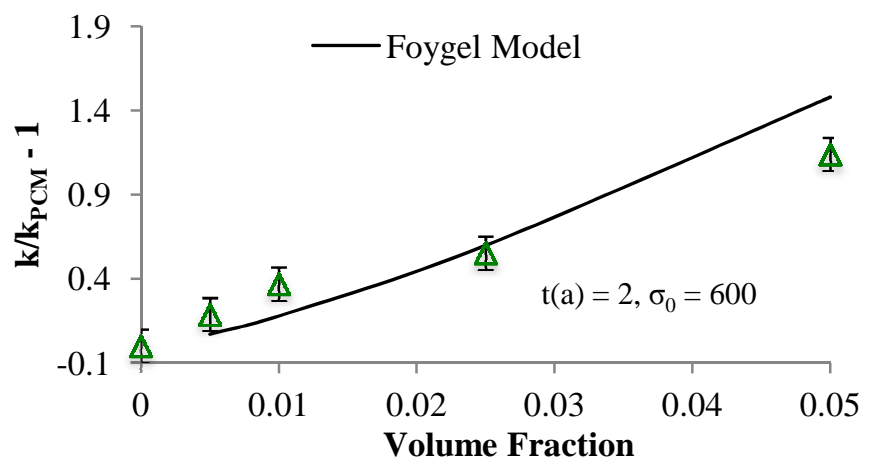

(c) $40 \mathrm{~nm}$ MWCNTs 


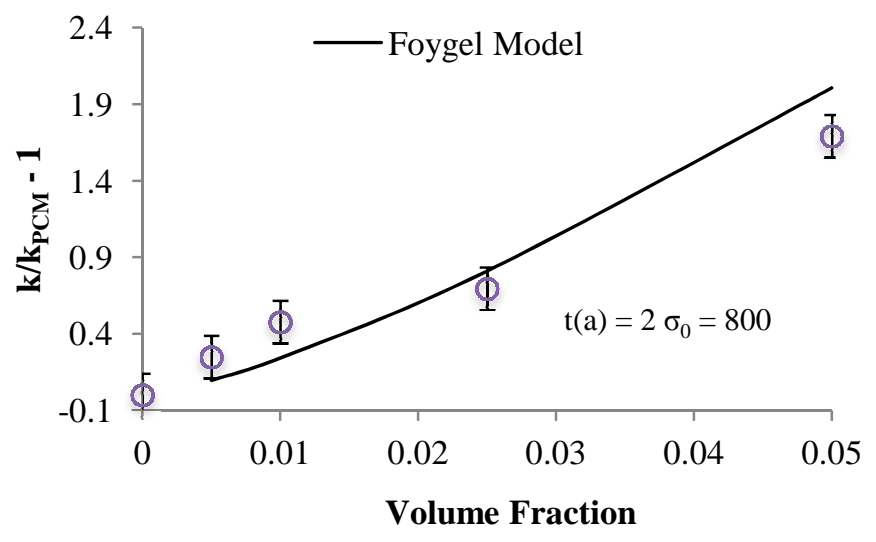

266 Fig. 2: Curve fits for: (a) MWCNTs with average diameter $=7 \mathrm{~nm}$, (b) MWCNTs with average 267 diameter $=10 \mathrm{~nm}$, (c) MWCNTs with average diameter $=40 \mathrm{~nm}$ and (d) MWCNTs with average diameter $=65 \mathrm{~nm}$ using the model developed by Foygel et al. [23]

270 According to the curve fits in Figs. 2 (a) - (d), the conductivity exponent (t(a)) is determined to 271 be $\sim 1.3$ for each of the distributions. While this value was previously calculated to be valid for 272 spherical nanoparticles [23], [27], it is expected that the nanoparticle networks first begin to 273 cluster into larger microspheres prior to percolation [28]-[31].

275 Using the curve fits that are calculated in Figs. 2 (a) - (d), the interfacial thermal resistance can 276 be calculated using Eqn. 5. The calculated values for interfacial thermal resistance between 277 individual MWCNTs within paraffin is given as a function of contact area in Fig. 3. 


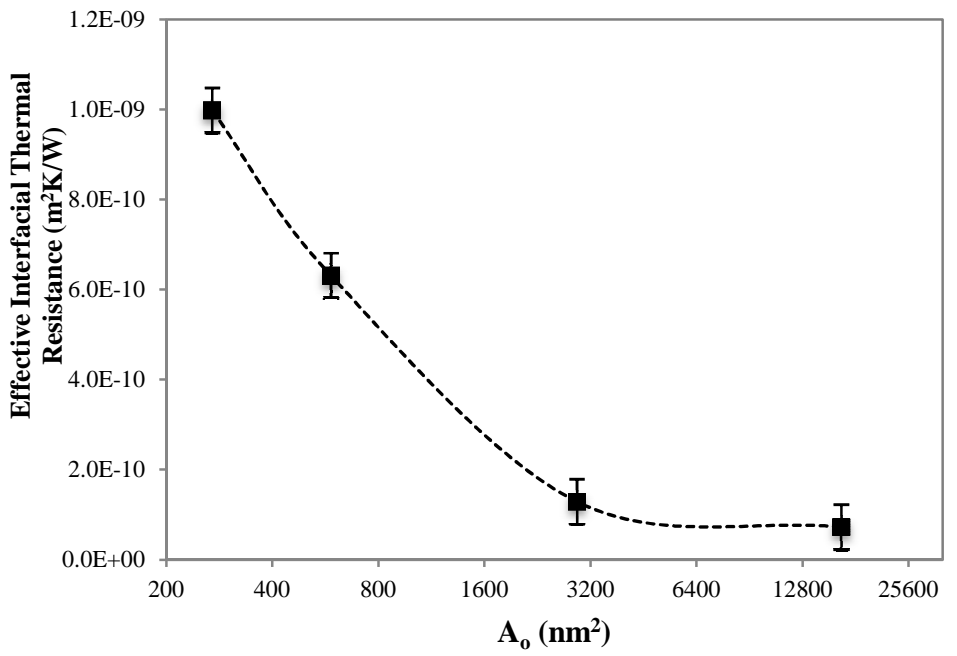

$280 \quad$ Fig. 3: Effective interfacial thermal resistance at MWCNT-MWCNT junctions as a function of overlap area

283 As shown in Fig. 3, the interfacial thermal resistance between contacting nanoparticles decreases 284 as a function of increasing contact area. Note that the dashed line in Fig. 3 does not constitute a 285 quantitative analysis of a trend between data points, but instead represents a qualitative 286 illustration of the data. In this figure, the interfacial thermal resistance is shown for calculations 287 made by both the Foygel at al. [23] and Wemhoff [32] models. Here, both models are in good 288 agreement with each other, which increases our confidence in the quantitative assessment of 289 interfacial thermal resistance given the different physics with which each model is constructed. 290 Interestingly, the effective interfacial thermal resistance decreases by a factor of $\sim 13$ as the 291 overlap area increases from $270 \mathrm{~nm}^{2}$ to $16,500 \mathrm{~nm}^{2}$. One possible explanation for this reduction 292 is the decrease in the ballistic contribution to interfacial thermal resistance at larger interfaces 293 that are formed between contacting CNTs. The relative contributions from ballistic and diffusive 294 transport across the interface can be determined using the analyses provided by Prasher [33]. 295 Both the diffusive and ballistic components of heat flow at the MWCNT/MWCNT interface can 296 be described by Eqns. 6 and 7.

$298 \quad R_{d}=\frac{1}{4 \cdot k_{p} \cdot b}$

299

$300 \quad R_{b}=\frac{4 l}{3 k_{p} A_{c}}$ 
In Eqn. 6, $\mathrm{b}$ represents the effective diameter of the contact area between individual nanoparticles $(\mathrm{nm}), \mathrm{A}_{\mathrm{c}}$ represents the contact area at the junction and 1 represents the mean free path of phonons $(\mathrm{nm})$ within each CNT. In the analysis provided by Wemhoff [24], the contact geometry is assumed to be rectangular. In this work, the contact geometry is assumed to be rectangular with equal dimensions (i.e. a square) for simplicity. Based on these assumptions, a nanoparticle-nanoparticle contact width can be extracted based on the analyses of Prasher [32] and Bahadur et al. [34] modified to fit the constraints of the contacting geometry encountered in this work. The calculated contact widths and areas are provided in Table 3. Additionally, the mean free path of phonons within each MWCNT as well as their effective, intrinsic thermal conductivities are calculated using the Wemhoff model [24] and also listed in Table 3.

\begin{tabular}{cccccc}
\hline $\begin{array}{c}\text { Diameter } \\
(\mathbf{n m})\end{array}$ & $\begin{array}{c}\text { Length } \\
(\boldsymbol{\mu m})\end{array}$ & $\begin{array}{c}\text { Contact } \\
\text { Width, } \\
\mathbf{b}(\mathbf{n m})\end{array}$ & $\begin{array}{c}\text { Contact } \\
\text { Area, } \mathbf{A}_{\mathbf{c}} \\
\left(\mathbf{p m}^{2}\right)\end{array}$ & $\begin{array}{c}\mathbf{k}_{\mathbf{p}} \\
(\mathbf{W} / \mathbf{m K})\end{array}$ & $\begin{array}{c}\lambda_{\mathbf{c}} \\
(\mathbf{n m})\end{array}$ \\
\hline \hline $\mathbf{7}$ & 20 & 0.016 & 81 & 32.8 & 4.4 \\
$\mathbf{1 0}$ & 50 & 0.022 & 169 & 44.2 & 8.2 \\
$\mathbf{2 5}$ & 15 & 0.038 & 225 & 69.1 & 13.0 \\
$\mathbf{6 5}$ & 15 & 0.098 & 1681 & 89.1 & 16.7
\end{tabular}

315 At first glance, the intrinsic thermal conductivity of each MWCNT appears to be low relative to 316 the majority of results presented in the literature [12]. However, the thermal conductivity of an $317 \mathrm{sp}^{2}$ hybridized carbon nanoparticle is known to scale as $\mathrm{k} \sim \ln (\mathrm{n})$, where $\mathrm{n}$ is the number of 318 graphene layers that surround the inner/bottom-most graphene layer, when the nanoparticle rests 319 on (or is embedded within) a surrounding material [35]. In this configuration, the nanoparticle's 320 intrinsic thermal conductivity is also known to be lower than the value for bulk graphite until a 321 critical number of channels for thermal transport are available such that the value for the thermal 322 conductivity of bulk graphite is reached. Consequently, the values for thermal conductivity in 323 Table 3 are not entirely unexpected. The line contact widths in Table 3 are calculated using the 324 diameter-dependent bending moduli supplied by Poncharal et al [36]. Additionally, the 325 interaction energy between the two cylindrical nanoparticles is assumed to take the following 326 form:

$$
U(l)=-\frac{3}{8} \pi \cdot A_{h} \cdot \frac{R^{4}}{(2 R+l)^{5}} \cdot\left[1+\frac{25 R^{2}}{(2 R+l)^{2}}\right]
$$


330 In Eqn. 8, $\mathrm{A}_{\mathrm{h}}$ is the Hamaker Constant, 1 is the separation distance between the nanotubes $(0.6$

$331 \mathrm{~nm}[22])$ and $\mathrm{R}$ is the radius of the nanotube (D/2). To find the van der Waals force exerted by

332 one MWCNT on the other, Eqn. 8 is differentiated with respect to the separation distance, 1. The

333 line contact width is subsequently calculated using Eqn. 9.

$335 \quad b=\frac{1}{2} \cdot\left(\frac{16 \cdot F_{v d w} \cdot E_{m} \cdot D}{\pi}\right)^{1 / 2}$

337 Given that the mean free path of phonons $(\lambda)$ in $\mathrm{sp}^{2}$ hybridized carbon is on the order of microns

338 [35] (and that the lowest mean free path in MWCNTs found experimentally to date is $20 \mathrm{~nm}$ ), 339 energy carriers are expected to traverse across MWCNT contacts ballistically (i.e. $\lambda>>$ b). Thus, 340 the primary constriction resistance $\left(R_{\text {constriction }}\right)$ and thermal conductance $\left(G=1 / R_{\text {constriction }}\right)$ can be 341 calculated using Eqn. 7. The thermal conductance at the contacting region $(\mathrm{G})$ is shown as a 342 function of contact area $\left(\mathrm{A}_{\mathrm{c}}\right)$ in Fig. 4.

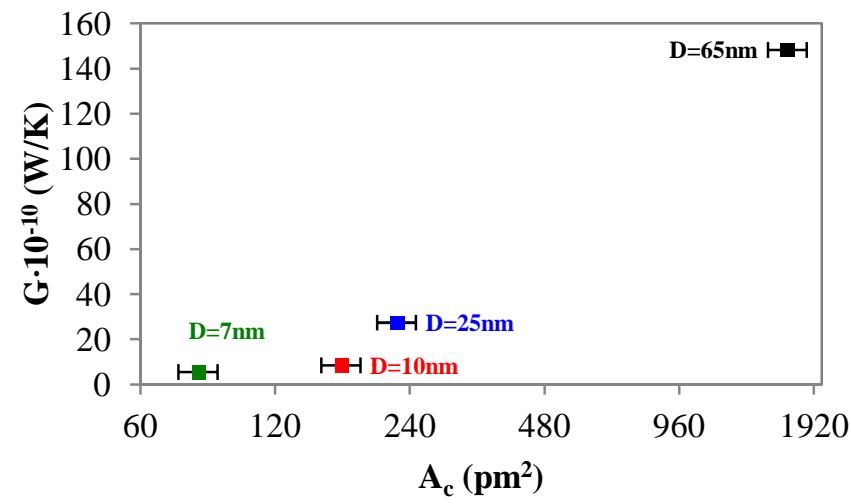

Fig. 4: Thermal conductance at MWCNT/MWCNT contacts as a function of contact area

347 Figure 4 reveals that the thermal conductance (that is, the amount of heat that can traverse 348 through the nanoparticle contact) increases by a factor of 27 as the contact area between 349 MWCNTs increases from $81 \mathrm{pm}^{2}$ to $1681 \mathrm{pm}^{2}$.

351 As a consequence of these physical phenomena, fundamental alterations to the adhesion energy 352 (i.e. the strength of the bond between nano materials) must be improved in the future if thermal 353 engineers are to further increase the thermal conductance across CNT interfaces. 
354 Recommendations for future research in this area include the application of wrapped polymers

355 [37] and atomic layer deposition (ALD) treatments [38] at the nanoparticle interfaces in order to

356 strengthen the chemical bonding between individual, contacting nanoparticles. This is expected

357 to allow engineers to move toward the full utilization of the unique physics of nanoparticles in 358 macroscopic applications. Additionally, it will also allow scientists and engineers to determine 359 the relative contributions of the adhesion energy and contact area between touching 360 nanostructures on the heat flow between them when used in conjunction with the work done in 361 this study.

\section{Conclusions}

364 In this study, MWCNTs with different diameters are used to produce percolating nanocomposite 365 phase change materials (PCMs). It was found that nanoparticles with smaller diameters produce 366 PCMs with lower thermal conductivities than nanoparticles with larger diameters. This is in 367 contrast to predictions that can be made with effective medium approximations when it is 368 assumed that nanoparticles with smaller diameters have higher thermal conductivities. Using 369 microscale analytical techniques, it is determined that the magnitude of impeded heat flow at the 370 junction increases as a function of decreasing contact area. This is found to be due to the increase 371 in ballistic phonon scattering at the interface as the contact area between nanoparticles decreases.

372 Consequently, the contact area between nanoparticles is found to play a significant role in heat 373 flow at the microscale, resulting in a significant augmentation in the bulk thermal conductivity of 374 percolating nanocomposite PCMs.

\section{Acknowledgements}

377 The authors appreciate the support of the National Science Foundation (CBET1235769) and the 378 Environmental Protection Agency Science to Achieve Results (STAR) Fellowship. Any opinions, 379 findings, and conclusions or recommendations expressed in this material are those of the 380 author(s) and do not necessarily reflect the views of the National Science Foundation. The 381 research described in this paper has also been funded in part by the United States Environmental 382 Protection Agency (EPA) under the Science to Achieve Results (STAR) Graduate Fellowship 383 Program. EPA has not officially endorsed this publication and the views expressed herein may 384 not reflect the views of the EPA. 
387 [1] M. J. Vesligaj and C. H. Amon, "Transient thermal management of temperature

[2] P. Goli, S. Legedza, A. Dhar, R. Salgado, J. Renteria, and A. A. Balandin, "Grapheneenhanced hybrid phase change materials for thermal management of Li-ion batteries," $J$. Power Sources, vol. 248, pp. 37-43, Feb. 2014.

[3] S. Pincemin, X. Py, R. Olives, M. Christ, and O. Oettinger, "Elaboration of Conductive Thermal Storage Composites Made of Phase Change Materials and Graphite for Solar Plant," J. Sol. Energy Eng., vol. 130, no. 1, p. 011005, Feb. 2008.

[4] K. Nithyanandam, R. Pitchumani, and A. Mathur, "Analysis of a latent thermocline storage system with encapsulated phase change materials for concentrating solar power," Appl. Energy, vol. 113, pp. 1446-1460, 2014.

[5] P. Charvát, L. Klimeš, and M. Ostrý, "Numerical and experimental investigation of a PCM-based thermal storage unit for solar air systems," Energy Build., vol. 68, pp. 488497, 2014.

[6] R. J. Warzoha, R. M. Weigand, and A. S. Fleischer, "Temperature-dependent thermal properties of a paraffin phase change material saturated with herringbone style graphite nanofibers," Appl. Energy, p. In Press, 2014.

[7] K. Chintakrinda, R. J. Warzoha, R. D. Weinstein, and A. S. Fleischer, "Quantification of the Impact of Embedded Graphite Nanofibers on the Transient Thermal Response of Paraffin Phase Change Material Exposed to High Heat Fluxes," J. Heat Transfer, vol. 134, no. 7, p. 071901, 2012.

[8] O. Sanusi, R. Warzoha, and A. S. Fleischer, "Energy storage and solidification of paraffin phase change material embedded with graphite nanofibers," Int. J. Heat Mass Transf., vol. 54, no. 19, pp. 4429-4436, 2011.

[9] R. J. Warzoha, D. Zhang, G. Feng, and A. S. Fleischer, "Engineering interfaces in carbon nanostructured mats for the creation of energy efficient thermal interface materials," Carbon, vol. 61, pp. 441-457, Sep. 2013.

[10] O. Sanusi, R. Warzoha, and A. S. Fleischer, "Energy storage and solidification of paraffin phase change material embedded with graphite nanofibers," Int. J. Heat Mass Transf., vol. 54, no. 19-20, pp. 4429-4436, Sep. 2011.

[11] E. Pop, D. Mann, Q. Wang, K. Goodson, and H. Dai, "Thermal conductance of an individual single-wall carbon nanotube above room temperature.," Nano Lett., vol. 6, no. 1, pp. 96-100, Jan. 2006. 
[12] A. M. Marconnet, M. A. Panzer, and K. E. Goodson, "Thermal conduction phenomena in carbon nanotubes and related nanostructured materials," Rev. Mod. Phys., vol. 85, no. 3, pp. 1295-1326, Aug. 2013.

[13] A. A. Balandin, "Thermal properties of graphene and nanostructured carbon materials.," Nat. Mater., vol. 10, no. 8, pp. 569-81, Aug. 2011.

[14] M. Koch, F. Ample, C. Joachim, and L. Grill, "Voltage-dependent conductance of a single graphene nanoribbon.," Nat. Nanotechnol., vol. 7, no. 11, pp. 713-7, Nov. 2012.

[15] R. J. Warzoha and A. S. Fleischer, "Heat Flow at Nanoparticle Interfaces," Nano Energy, vol. 6, pp. 137-158, 2014.

[16] H. Babaei, P. Keblinski, and J. M. Khodadadi, "Thermal conductivity enhancement of paraffins by increasing the alignment of molecules through adding CNT/graphene," Int. J. Heat Mass Transf., vol. 58, no. 1, pp. 209-216, 2013.

[17] H. Ji, D. P. Sellan, M. T. Pettes, X. Kong, J. Ji, L. Shi, and R. S. Ruoff, "Enhanced thermal conductivity of phase change materials with ultrathin-graphite foams for thermal energy storage," Energy Environ. Sci., vol. 7, pp. 1185-1192, 2014.

[18] M. Bozlar, D. He, J. Bai, Y. Chalopin, N. Mingo, and S. Volz, "Carbon nanotube microarchitectures for enhanced thermal conduction at ultralow mass fraction in polymer composites.," Adv. Mater., vol. 22, no. 14, pp. 1654-8, Apr. 2010.

[19] A. Yu, P. Ramesh, X. Sun, E. Bekyarova, M. E. Itkis, and R. C. Haddon, "Enhanced Thermal Conductivity in a Hybrid Graphite Nanoplatelet - Carbon Nanotube Filler for Epoxy Composites," Adv. Mater., vol. 20, no. 24, pp. 4740-4744, Dec. 2008.

[20] H. Sun, Z. Xu, and C. Gao, "Multifunctional, ultra-flyweight, synergistically assembled carbon aerogels.," Adv. Mater., vol. 25, no. 18, pp. 2554-60, May 2013.

[21] Z. Xu and M. J. Buehler, "Nanoengineering heat transfer performance at carbon nanotube interfaces.," ACS Nano, vol. 3, no. 9, pp. 2767-2775, 2009.

[22] H. Zhong and J. Lukes, "Interfacial thermal resistance between carbon nanotubes: Molecular dynamics simulations and analytical thermal modeling," Phys. Rev. B, vol. 74, no. 12, p. 125403, Sep. 2006.

[23] M. Foygel, R. Morris, D. Anez, S. French, and V. Sobolev, "Theoretical and computational studies of carbon nanotube composites and suspensions: Electrical and thermal conductivity," Phys. Rev. B, vol. 71, no. 10, p. 104201, Mar. 2005.

[24] A. P. Wemhoff, "Thermal conductivity predictions of composites containing percolated networks of uniform cylindrical inclusions," Int. J. Heat Mass Transf., vol. 62, pp. 255262, Jul. 2013. 
[25] R. J. Warzoha and A. S. Fleischer, "Determining the thermal conductivity of liquids using the transient hot disk method. Part I: Establishing transient thermal-fluid constraints," Int. J. Heat Mass Transf., vol. 71, pp. 779-789, Nov. 2014.

[26] V. Bohac, M. K. Gustavsson, L. Kubicar, and S. E. Gustafsson, "Parameter estimations for measurements of thermal transport properties with the hot disk thermal constants analyzer," Rev. Sci. Instrum., vol. 71, no. 6, p. 2452, 2000.

[27] R. Haggenmueller, C. Guthy, J. R. Lukes, J. E. Fischer, and K. I. Winey, "Single Wall Carbon Nanotube/Polyethylene Nanocomposites: Thermal and Electrical Conductivity," Macromolecules, vol. 40, no. 7, pp. 2417-2421, Apr. 2007.

[28] J. W. Gao, R. T. Zheng, H. Ohtani, D. S. Zhu, and G. Chen, "Experimental investigation of heat conduction mechanisms in nanofluids. Clue on clustering.," Nano Lett., vol. 9, no. 12, pp. 4128-32, Dec. 2009.

[29] W. Evans, R. Prasher, J. Fish, P. Meakin, P. Phelan, and P. Keblinski, "Effect of aggregation and interfacial thermal resistance on thermal conductivity of nanocomposites and colloidal nanofluids," Int. J. Heat Mass Transf., vol. 51, no. 5, pp. 1431-1438, 2008.

[30] R. Zheng, J. Gao, J. Wang, S.-P. Feng, H. Ohtani, J. Wang, and G. Chen, "Thermal percolation in stable graphite suspensions.," Nano Lett., vol. 12, no. 1, pp. 188-92, Jan. 2012.

[31] J. J. Wang, R. T. Zheng, J. W. Gao, and G. Chen, "Heat conduction mechanisms in nanofluids and suspensions," Nano Today, vol. 7, no. 2, pp. 124-136, 2012.

[32] A. P. Wemhoff, "Thermal conductivity predictions of composites containing percolated networks of uniform cylindrical inclusions," Int. J. Heat Mass Transf., vol. 62, pp. 255262, 2013.

[33] R. Prasher, "Predicting the thermal resistance of nanosized constrictions," Nano Lett., vol. 5, no. 11, pp. 2155-9, Nov. 2005.

[34] V. Bahadur, J. Xu, Y. Liu, and T. S. Fisher, "Thermal Resistance of Nanowire-Plane Interfaces," J. Heat Transfer, vol. 127, no. 6, p. 664, Jun. 2005.

[35] E. Pop, V. Varshney, and A. K. Roy, "Thermal properties of graphene: Fundamentals and applications," MRS Bull., vol. 37, no. 12, pp. 1273-1281, Nov. 2012.

[36] P. Poncharal, "Electrostatic Deflections and Electromechanical Resonances of Carbon Nanotubes," Science (80), vol. 283, no. 5407, pp. 1513-1516, Mar. 1999.

[37] Z. Xu and M. J. Buehler, "Nanoengineering heat transfer performance at carbon nanotube interfaces.," ACS Nano, vol. 3, no. 9, pp. 2767-75, Sep. 2009.

[38] M. I. Dafinone, G. Feng, T. Brugarolas, K. E. Tettey, and D. Lee, "Mechanical reinforcement of nanoparticle thin films using atomic layer deposition.," ACS Nano, vol. 5, no. 6, pp. 5078-87, Jun. 2011. 